\title{
Smart Wearable Device for Elderly Dementia People
}

\author{
Surendran D, Kiruthika V R
}

\begin{abstract}
Millions of individuals worldwide are infected by a serious disorder known as Dementia with collection of symptoms which will embody blackout and feel inconvenient to think, solve problems. Dementia is controllable if the person is within the drafting board of not wellness, so as to help such dementia affected people, the target is to style sensible smart wearable device that motivates and enthusiast their life easier. The good device practises on 3 operations. At first the routine activities are monitored by the sensors and trained using a machine learning algorithms which are fed to a smart device based on the data, it guides the person with physical activities. Then an indoor tracking of a person is carried out by beacon signals through which if person forgets to do next activity, smart device guides them by learning the patterns of a person about their movements in indoor. At last an outdoor tracking of a person is carried out using GPS signals enabled in a smart device which helps the person to find their way to home even when they missed the way or suffers from memory loss. If any different texture or feedback is not received from the patient to any of the above mentioned activities the smart device alerts the caretaker in the form of message through app. Thus, this smart device reduces the burden of the caretaker
\end{abstract}

Keywords: Dementia, GPS, Beacon Signals, Machine Learning, Sensors, Problem Solving.

\section{INTRODUCTION}

Three in 10 individuals over the age of eighty-five and nearly one in 10 individuals over sixty-five have Dementia. Associate degree calculable 1.2 million individual's square measure concerned within the care of an individual with Dementia, Autism, and Alzheimer's all in common leads to cognitive thinking, difficulty to remember thinks which is very dangerous. Dementia affects millions of people including elder people who usually under prolonged monitoring and aided living, and without having much freedom. Dementia patients require constant assistance and it is difficult job for care takers to take care of such patients. Integrated care research for dementia is limited and focuses on applying existing information technologies in the care settings. Current solutions for helpful living for dementedness at typically part primarily based and standalone.

Revised Manuscript Received on March 16, 2020.

* Correspondence Author

Surendran D *, Computer Science and Engineering, Sri Krishna College of Engineering and Technology, Coimbatore, India. Email: surendran @skcet.ac.in

Kiruthika V.R, Computer Science and Engineering, Sri Krishna College of Engineering and Technology, Coimbatore, India.Email: 18epcs002@skcet.ac.in

(C) The Authors. Published by Blue Eyes Intelligence Engineering and Sciences Publication (BEIESP). This is an open access article under the CC BY-NC-ND license (http://creativecommons.org/licenses/by-nc-nd/4.0/)
The technologies are limited, proprietary, expensive and not flexible to be integrated and adapted to different environments, evolving needs and personal preferences. AI technologies improves and extends human cognitive capabilities can help prevent, diagnose and predict the conditions, symptoms which support the daily activities can be controlled, and optimize the work of care givers and treatment functions for dementia patients at home. Developing a wearable device which is based on machine learning and sensors to assist the dementia patients with several functions is difficult. There are many shortcomings are to be entangled while developing a user friendly wearable device in respect to the cost, flexible one. This product must be efficient and should be used by every dementia affected people in cases based on all aspects of structure, cheap and performance. This smart device comprised of three different functions with sensors, beacons etc which performs efficient to process data and track location in real time by consuming minimum power that extends battery life of the device. It manipulates the system's environment, computes and response with accurate results obtained in real time been transformed it to the caretaker as soon as it acquired.

\section{RELATED WORK}

The main objective of every navigation system is to navigate object's location in a given environment. This can be carried out and processed in many ways, and triangulation method [5] is one of them that work on referred object location. The measuring system while not moving components is less complicated to implement, energy-efficient and additional immune to disturbances like vibration. The work presents as in depth explanation of the static positioning system prototype. The systems complete processing starting from hardware to the machine learning algorithms at the end is fully explained and discussed. The experimental precision of the system has been analyzed and examined with excellent results - the accuracy is cherished by the moving components used in existing systems. The results not solely showed the correct work of the system, however additionally unconcealed facts that area unit predicted to improve the experimental precision of the device. The challenges of classifying and processing the routine activities are stated [1] with the deep learning algorithms using dataset. Usually these activities are processed by the Recurrent Neural Network that is defined with mean accuracy and classification results are compared [6] for various activities.

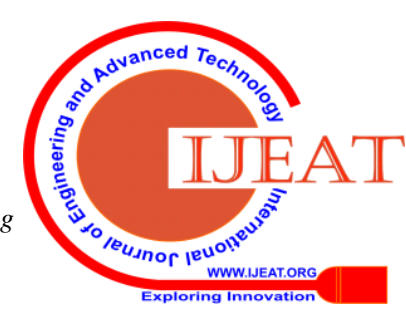


Conventional neural network is been used for extracting the feature from the activities that are monitored and executed using the LSTM algorithm units [2]. The use cases and various strategies of datasets are explained with open

\section{III.PROPOSED ARCHITECTURE}

Dementia is a neurodegenerative disorders that suffers from memory loss, cognitive decline which leads to inability in performing essential daily life activities. We express the matter of classifying the lifestyle behaviors of someone independent of sensing element relies mostly on monitored information. In addition to it, Indoor positioning of a person can be sensed continuously by the beacon signals. Recurrent neural network is used to track successive sensor data inputs. This work implements the assistive care for dementia people by reminding the routine activities and confident to live without the care taker.

The proposed system is to develop smart device for dementia patients which is used to monitor them and receive the data sensed by the sensor. The daily activities are learned and patterns can be predicted. Recurrent neural networks are used for classifying the routine activities by automatically extracting the feature process while the data been sequentially addressed. RFID tags are used to identify a specific person and ensure that the watch belong to the respective person.

This reduces the burden of caregivers from monitoring the patient throughout the day. The smart device which is used to monitor the routine activities of the person by using the sensors. To overcoming the use of cameras for indoor positioning, we use the beacons to track the person inside the home. For outdoor tracking, a GPS that are enabled in smart device are used to track the person outside. All these process are proposed to overcome the difficulties of a person as well as caregivers in the hospitals and home assisted patients. If the response is not received from the persons, an alert is send to the caregivers from the smart device.

The following Fig.1. explains the structure of smart wearable device for dementia people. The device consists of wireless modules such as Bluetooth for collecting the signals from the sensor so that an indoor positioning can be processed. The Wi-Fi can be used for greater accuracy from the signal as a combination of both Bluetooth and W-Fi signals. The GPS technology that is used for tracking outdoor if patient went outside.

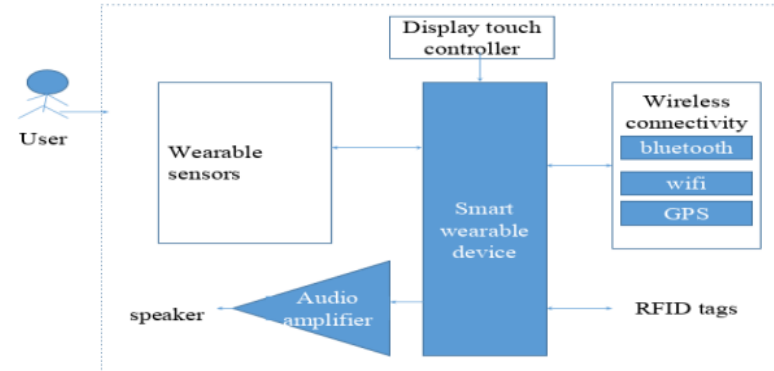

Fig. 1. Smart Wearable Device

The instructions can be guided by audio format that is from the audio amplifier with the help of speaker and wearable sensors are explained in the table 1. The RFID(Radio dataset of technology Bluetooth Low Energy (BLE) [9].The essential parameter of Elder dementia patients such as their needs and comfort from existing patients are studied for the development of smart homes [4]. Frequency Identification) tags are used to identify the right user receiving the Bluetooth signals by

unique id for every user in the surrounding. These signals are processed and analyze the routine activities and location of the user in the backend with which the device guides the user.

The wearable device should be comfortable for the patients so that they didn't feel sick and disturbed to wear it every day.

Table- I: Wearable Sensors and its Usage

\begin{tabular}{|c|l|c|}
\hline \multirow{2}{*}{ Sr. No. } & \multicolumn{2}{|c|}{ Wearable Sensors } \\
\cline { 2 - 3 } & \multicolumn{1}{|c|}{ Sensors } & Usage \\
\hline 1 & Accelerometer & $\begin{array}{c}\text { Direction } \\
\text { of gravity }\end{array}$ \\
\hline 2 & Gyroscope & $\begin{array}{c}\text { Angular } \\
\text { position } \\
\text { and rotation }\end{array}$ \\
\hline 3 & Heart rate Sensor & $\begin{array}{c}\text { Measures a } \\
\text { heart rate }\end{array}$ \\
\hline 4 & Blood Pressure Sensor & $\begin{array}{c}\text { Measure } \\
\text { blood } \\
\text { pressure }\end{array}$ \\
\hline
\end{tabular}

In Table.1, it explains the wearable sensors and its various usages according to the sensor that are fixed in the smart device for efficient health monitoring. The beacons are fixed at the walls for tracking the person indoor via Bluetooth signals transferred to the device.

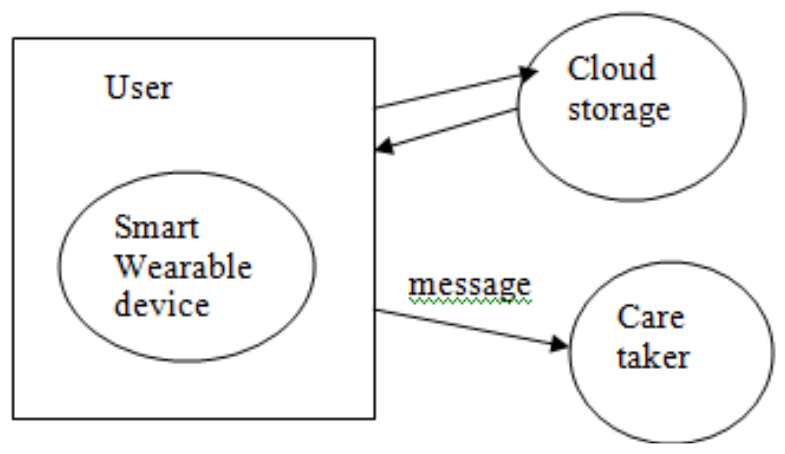

Fig. 2. Communication Diagram

The device communicates with the caretaker through message if there is no feedback from the respective patient. All the details such as signals received from the beacons, heart rate, accelerometer measurements, blood pressure rate and gyroscope readings are stored in cloud for further processing the data. Later the processed data been sent to the device with the correct audio preferred one to guide the patients. This is defined in fig 2.

\section{IV.MODULE DESCRIPTION}

There are three main modules that are analyzed and executed for developing a wearable smart device for dementia people to overcome the existing problems are

Published By:

Blue Eyes Intelligence Engineering 

A. Monitoring routine activities
B. Indoor tracking of a person
C. Outdoor tracking of a person

\section{A. Monitoring Routine Activities:}

The data of routine activities of the person was gathered with measurement units such as accelerometer, gyroscope and a pulse sensor. These measuring sensors were positioned at different areas of body such as chest, right wrist and right ankle for accuracy. The data sets are constructed into two and were used; first one with routine activities and the second one with body movements monitored from daily life activities. Routine activities are acquired from sensors positioned at various regions of the body were used, meanwhile the body movements are gathered from the accelerometer and gyroscopes were used. The daily life activities are monitored using a wearable sensors which indicates an objective measure of activity levels or restrictions that are allotted for patients.

\section{B. Indoor Tracking of a Person:}

Indoor positioning of people with beacons provides decisive advantages for projects that are hooked into high accuracy and flexible to all devices (with different OS).Beacon transmitters are placed at several positions within the building. The signals can be transmitted via Bluetooth to wearable devices of patients. Like that, it is possible to track their position continuously and transmit it to the indoor navigation system. An app installed on the smart device interprets the signals.

\section{Outdoor Tracking of a Person:}

Outdoor navigation includes the location can be tracked of a mobile object or person are usually carried out using international. The GPS unit consumes much more power than any other sensors in a smart device. This wearable device which consumes low power and low cost of sensors used to navigate locations of a moving person continuously with great accuracy. The smart wearable device contains sensors such as accelerometer, the magnetometer and the gyroscope has been fixed inbuilt and utilized, rather than using costly GPS unit, to navigate the continual locations of a patients.

\section{WORKING OF SMART WEARABLE DEVICE}

The steps involved to develop a smart wearable device for monitoring and assisting the elderly dementia people are

A. Data collection from various sources

B. Pre-process the data

C. Train the data using machine learning algorithms

\section{A. Data collection from various sources:}

The daily life activities are sensed and data retrieved from the sensors such as accelerometer, gyroscope and classify the data using machine learning algorithms.

An indoor tracking of a person data collected from the beacon signals that are fixed on the wall of every room in a home and their pattern is predicted.

An outdoor tracking of a person data collected from

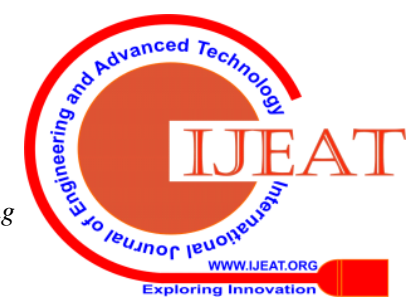

the GPS data that are stored on the smart device.

Data pre-processing involves reworking data into an obvious format. Real-world information is usually not complete (missing values), inconsistent, and is probably going to tain several flaws in data. Information pre-processing is technique of breaking down such problematic can be reduction.

\section{Train the data using machine learning algorithms:}

Classification Algorithms in machine learning such as knearest neighbor and decision trees are the classifiers were used to classify the activities and results been obtained for decision trees, with an accuracy of $84 \%$. Various classification algorithms: Classification and Regression Trees, k-Nearest Neighbor and Support Vector Machine were executed. The advantage of our approach extracts the feature from the data while the sensors data which explained

Table- II: Comparison of Classifiers

\begin{tabular}{|l|c|c|}
\hline \multirow{2}{*}{ Parameters } & \multicolumn{2}{|c|}{ Classifiers for Routine Activities } \\
\cline { 2 - 3 } & Knn & SVM \\
\hline Accuracy score & 0.9424603174603174 & 0.9444444444444444 \\
\hline Precision score & 0.9407144585953463 & 0.9469968113750296 \\
\hline Recall score & 0.9471771683850795 & 0.9476696511756989 \\
\hline F1 score & 0.942958400926144 & 0.9451762492796217 \\
\hline
\end{tabular}

Two different classifiers such as Support Vector Machine SVM),K nearest neighbor(Knn) are used to classify the set without any outliers which produce variation in the explained in Table 2. This comparison results nearly

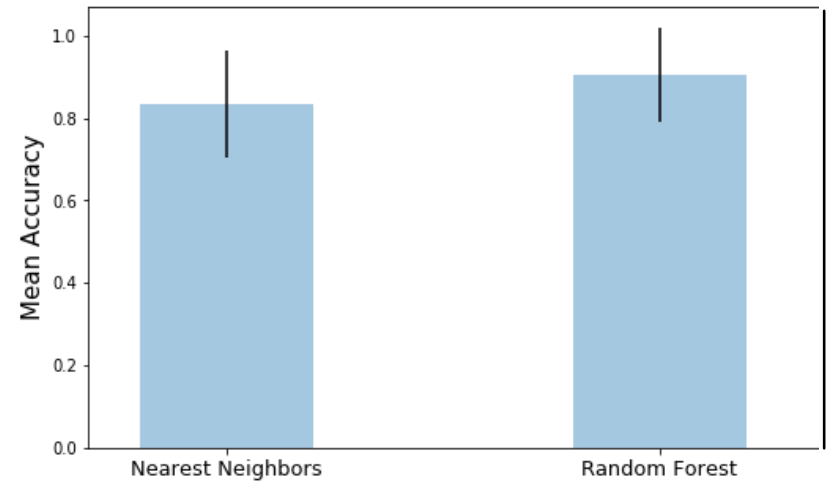

Fig. 3. Classifiers of Indoor Tracking

The classifiers for indoor tracking such as Nearest Neighbor and Random Forest are executed for classifying the indoor dataset processed from the readings of beacon signals is explained in Fig 3 with the mean accuracy values. 
Table- III: Comparison of Classifiers

\begin{tabular}{|c|c|c|}
\hline \multirow{2}{*}{ Parameters } & \multicolumn{2}{|c|}{ Classifiers for Indoor Tracking } \\
\cline { 2 - 3 } & $\begin{array}{c}\text { Nearest } \\
\text { Neighbor }\end{array}$ & Random Forest \\
\hline $\begin{array}{c}\text { Mean } \\
\text { Accuracy }\end{array}$ & 0.8339466089466089 & 0.9041847041847042 \\
\hline
\end{tabular}

The Mean Accuracy of classifiers that are used to classify the indoor tracking dataset has a higher accuracy for the Random Forest as $90 \%$ compared to Nearest Neighbor is explained in table 3.

\section{RESULT}

The results been processed for the routine activities using the sensors and indoor positioning by the beacons respectively.

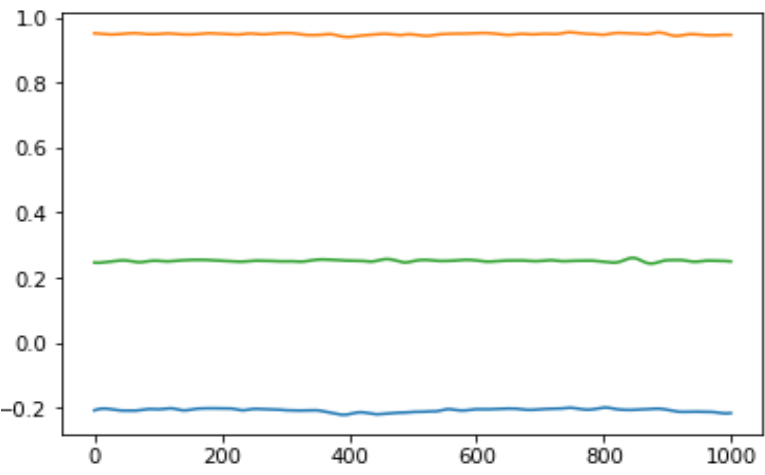

Fig. 3. Activity detection graph

The activity of the dementia patient is recorded by the sensor called accelerometer is analysed and the readings are filtered using ensemble filter which is showed in Fig. 3 with the colour as orange, green and blue defines the motion of the patient at different measure.

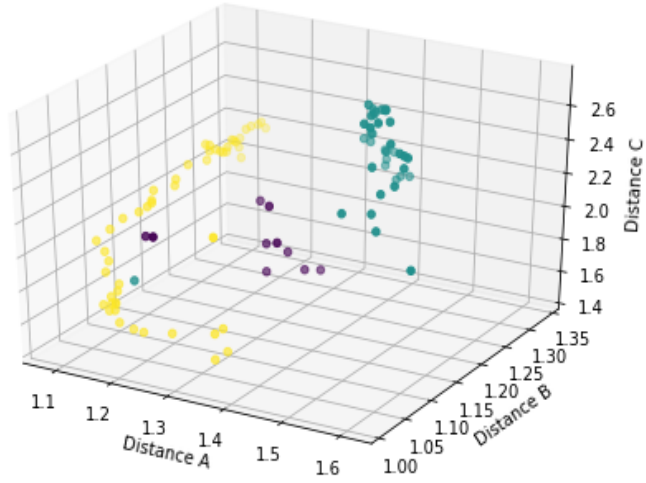

Fig. 4. 3D axes of indoor location

The indoor locations are analyzed by three distances such as distance $\mathrm{A}$, distance $\mathrm{B}$, distance $\mathrm{C}$ are located in 3D axes of axes 1 as yellow color in distance $A$, axes 2 as purple in distance $\mathrm{B}$ and axes 3 as green in distance $\mathrm{C}$ are plotted for the respective beacons in a surrounding of the patients.

\section{CONCLUSION}

Smart wearable device is proposed to monitor and classify the entire activities of a dementia persons and indoor tracking of a person is carried out by beacon signals which is trained and pattern in which the patient move are predicted by the algorithms such as support vector machine, k Nearest Neighbor algorithm and Random Forest for classifying data and processed it to produce the results with promising mean accuracy. If these are not worked properly, the smart device alerts the caregiver of a dementia affected person through message. It provides the pattern of a location that is visited by the dementia person with time series data. RFID tags are used to identify the person who wears the smart device in order to prevent other users to wear the device.

\section{FUTURE WORK}

Future work of this project, in order the reduce the burdens of caretaker as well as make this product efficient is to ensure the user continuously wears the device to prevent from missing the device anywhere on their way and a web application as well as mobile app is to develop for tracking the patient real time by the caretaker using their device. This provides an efficient working of the system for the patients.

\section{REFERENCES}

1. Henry Friday Nwekea, Ying Wah Teha, Mohammed Ali Al-garadi, Uzoma Rita Alo," Deep learning algorithms for human activity recognition using mobile and wearable sensor networks: State of the art and research challenges"

2. Francisco Javier Ordóñez and Daniel Roggen," Deep Convolutional and LSTM Recurrent Neural Networks for Multimodal Wearable Activity Recognition"

3. Parisa Rashidi, Member, IEEE, and Alex Mihailidis" A Survey on Ambient-Assisted Living Tools for Older Adults"

4. Deepika Singh1(B), Johannes Kropf1, Sten Hanke1, and Andreas Holzinger2," Ambient Assisted Living Technologies from the Perspectives of Older People and Professionals"

5. Maciej Ciężkowski," A prototype of static IR beacon-receiver positioning system based on triangulation method"

6. Roxana Jurca, Tudor Cioara, Ionut Anghel, Marcel Antal, Claudia Pop, Dorin Moldovan," Activities of Daily Living Classification using Recurrent Neural Networks"

7. Shirin Enshaeifar, Payam Barnaghi, Severin Skillman, Andreas Markides, Tarek Elsaleh, Sahr Thomas Acton, Ramin Nilforooshan, Helen Rostill," The Internet of Things for Dementia Care"

8. Óscar Belmonte-Fernández, Adrian Puertas-Cabedo, Joaquín TorresSospedra, Raúl Montoliu-Colás and Sergi Trilles-Oliver "An Indoor Positioning System Based on Wearable's for Ambient-Assisted Living"

9. Paolo Baronti, Paolo Barsocchi, Stefano Chessa, Fabio Mavilia and Filippo Palumbo "Indoor Bluetooth Low Energy Dataset for Localization, Tracking, Occupancy, and Social Interaction"

10. Yida Zhu, ${ }^{1}$ Haiyong Luo, ${ }^{2, *} \mathrm{Qu}$ Wang, ${ }^{3}$ Fang Zhao, ${ }^{1, *}$ Bokun Ning, ${ }^{1}$ Qixue Ke, ${ }^{1}$ and Chen Zhang ${ }^{1}$ "A Fast Indoor/Outdoor Transition Detection Algorithm Based on Machine Learning”.

\section{AUTHORS PROFILE}

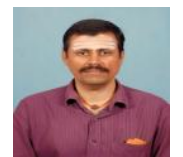

D. Surendran, has 15 years of experience in teaching UG and PG Computer Science and Engineering and currently working as Professor in the Department of CSE, Sri Krishna College of Engineering and Technology. He obtained PG in Computer Science and Engineering and Ph.D in Information and Communication Engineering from Anna University. Research interests include Cloud Computing, Semantic Technologies, IoT. He has guided 3 Research scholars for their $\mathrm{PhD}$ in CSE.

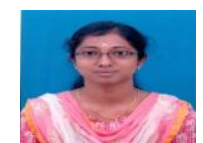

V.R. Kiruthika, is currently pursuing master degree of computer science and engineering in Sri Krishna College of Engineering and Technology in the year of 2020. She received UG degree in CSE from Sasurie College of Engineering. She attended more number of conferences and presented a paper in the field of computer science including machine learning, cloud. Her Area of Interest is Machine Learning and cloud. 